\title{
MODULAR CURVES OF D-ELLIPTIC SHEAVES ARE ASYMPTOTICALLY OPTIMAL
}

\author{
MiHRAN PAPIKIAN
}

\begin{abstract}
We prove that the series of modular curves of $\mathcal{D}$-elliptic sheaves with appropriate level structures attain the Drinfeld-Vladut bound over $\mathbb{F}_{q^{2}}$.
\end{abstract}

\section{Introduction}

1.1. Motivation. Let $q$ be a power of a prime $p$ and let $\mathbb{F}_{q}$ denote the finite field with $q$ elements. Let $\left\{C_{i}\right\}_{i \in \mathbb{N}}$ be a series of smooth, projective, geometrically connected curves defined over $\mathbb{F}_{q}$. Denote the genus of $C_{i}$ by $g_{i}$, and the set of $\mathbb{F}_{q}$-rational points on $C_{i}$ by $C_{i}\left(\mathbb{F}_{q}\right)$. Assume $g_{i} \rightarrow \infty$ as $i \rightarrow \infty$. A well-known result of Drinfeld and Vladut [14] says that

$$
\limsup _{i \rightarrow \infty}\left(\frac{\# C_{i}\left(\mathbb{F}_{q}\right)}{g_{i}}\right) \leq \sqrt{q}-1
$$

This result, proven in the early 80's, was a significant and a rather surprising improvement of Weil's celebrated estimate from the 40's

$$
\# C\left(\mathbb{F}_{q}\right) \leq q+1+2 g \sqrt{q}
$$

in the case when the genus $g$ of $C$ is very large compared to $q$.

The series $\left\{C_{i}\right\}_{i \in \mathbb{N}}$ is called asymptotically optimal if

$$
\lim _{i \rightarrow \infty}\left(\frac{\# C_{i}\left(\mathbb{F}_{q}\right)}{g_{i}}\right)=\sqrt{q}-1
$$

or, in other words, $\left\{C_{i}\right\}_{i \in \mathbb{N}}$ realizes the bound (1.1). Asymptotically optimal series are important in coding theory via Goppa codes, and they are also quite interesting from arithmetic point of view.

If $q$ is not a square then no asymptotically optimal series of curves is known. If $q$ is a square then asymptotically optimal series always exist, but every known such series has the property that for all sufficiently large $i$ the curve $C_{i}$ is of one of the following types (see [7], [13], [10], [5]):

- classical modular curve, i.e., a curve classifying elliptic curves with some level structure, such as $X_{0}(N) / \mathbb{F}_{p^{2}},(N, p)=1$ (in this case $q=p^{2}$ );

- Shimura curve;

- Drinfeld modular curve.

Received by the editors March 30, 2007.

2000 Mathematics Subject Classification. Primary 11G18, 11G20; Secondary 11G09, 14G05.

Key words and phrases. Modular curves, Drinfeld-Vladut bound. 
(It is conjectured by Elkies [4] that asymptotically optimal series arising from certain recursive constructions always consist of modular curves.)

The purpose of this article is to expand the previous list by including the modular curves of $\mathcal{D}$-elliptic sheaves, which were introduced by Laumon, Rapoport and Stuhler in [9]. We show that the modular curves of $\mathcal{D}$-elliptic sheaves with appropriate level structures produce asymptotically optimal series of curves over $\mathbb{F}_{q}$, when $q$ is a square.

Just like Drinfeld modular curves are the function field analogues of classical modular curves over $\mathbb{Q}$, the modular curves of $\mathcal{D}$-elliptic sheaves are the function field analogues of Shimura curves, hence our result represents a natural final entry in the above list.

1.2. Main theorem. Before stating precisely the main result, we need to introduce some terminology and notation.

Let $X:=\mathbb{P}_{\mathbb{F}_{q}}^{1}$ be the projective line over $\mathbb{F}_{q}$. Denote by $F=\mathbb{F}_{q}(T)$ the field of rational functions on $X$. Fix a 4-dimensional central division algebra $D$ over $F$ (i.e., a quaternion algebra over $F$ ), which is split at $\infty=1 / T$. Let $R$ be the set of places where $D$ is ramified ${ }^{1}$. Fix a maximal order $\mathcal{D}$ in $D$ in the sense of $\S 2.2$. Let $o$ be a fixed closed point on $X-R-\infty$. Denote the residue field at $o$ by $\mathbb{F}_{o}$ and $q_{o}:=\# \mathbb{F}_{o}$. For each closed subscheme $I$ of $X$ such that $I \cap(R \cup o \cup \infty)=\emptyset$, let $\mathcal{E} \ell \ell_{I, o}$ be the modular scheme classifying $\mathcal{D}$-elliptic sheaves with zero $o$ and pole $\infty$, equipped with level- $I$ structures (see $\S 3$ for the definitions). Then $\mathcal{E} \ell \ell_{I, o}$ is a smooth, projective, 1-dimensional scheme over $\mathbb{F}_{o}$. Let $\mathcal{I}$ be the ideal sheaf of $I$, and denote $\mathcal{O}_{I}:=\mathcal{O}_{X} / \mathcal{I}, \operatorname{deg}(I):=\operatorname{dim}_{\mathbb{F}_{q}}\left(\mathcal{O}_{I}\right)$. The group $\mathrm{GL}_{2}\left(\mathcal{O}_{I}\right)$ acts naturally on $\mathcal{E} \ell_{I, o}$, by acting on the level structures. Let $H$ be a subgroup of $\mathrm{GL}_{2}\left(\mathcal{O}_{I}\right)$. Denote by $\mathcal{E} \ell \ell_{I, o}^{H}$ the quotient $\mathcal{E} \ell \ell_{I, o} / H$. Denote by $\mathbb{F}_{o}^{(2)}$ the quadratic extension of $\mathbb{F}_{o}$. Let $Z$ and $B$ denote the center and the Borel subgroup of $\mathrm{GL}_{2}$, respectively. Finally, decompose $\mathcal{I}=\mathfrak{p}_{1}^{s_{1}} \cdots \mathfrak{p}_{n}^{s_{n}}$ into a product of prime powers in the Dedekind domain $\mathbb{F}_{q}[T]$ and embed $\mathbb{F}_{q}^{\times}$diagonally into $\mathcal{O}_{I}^{\times}=\left(\mathcal{O}_{X} / \mathfrak{p}_{1}^{s_{1}}\right)^{\times} \times \cdots \times\left(\mathcal{O}_{X} / \mathfrak{p}_{n}^{s_{n}}\right)^{\times}$. Denote the quotient by $\mathcal{O}_{I}^{\times} / \mathbb{F}_{q}^{\times}$.

Theorem 1.1. Let $\left\{I_{i}\right\}_{i \in \mathbb{N}}$ be a sequence of closed subschemes of $X-R-\infty-o$, such that $\operatorname{deg}\left(I_{i}\right) \rightarrow \infty$. For each $i$ choose a subgroup $H_{i}$ of $\mathrm{GL}_{2}\left(\mathcal{O}_{I_{i}}\right)$ such that

(i) $Z\left(\mathcal{O}_{I_{i}}\right) \subseteq H_{i} \subseteq B\left(\mathcal{O}_{I_{i}}\right)$;

(ii) $\operatorname{det}\left(H_{i}\right)$ generates $\mathcal{O}_{I_{i}}^{\times} / \mathbb{F}_{q}^{\times}$.

Let $X_{i}:=\mathcal{E} \ell \ell_{I_{i}, o}^{H_{i}}$. Then $\left\{X_{i}\right\}_{i \in \mathbb{N}}$ is a sequence of projective, smooth, geometrically connected curves defined over $\mathbb{F}_{o}^{(2)}$ which is asymptotically optimal.

Example 1.2. To satisfy the conditions of the theorem, we can take $\left\{I_{i}\right\}$ to be all closed subschemes of $X-R-\infty-o$ (not necessarily reduced), arranged by their degrees, and $H_{i}=B\left(\mathcal{O}_{I_{i}}\right)$. Then $X_{i}$ 's are the analogues of classical modular curves with Hecke level structures.

Another choice which satisfies the conditions of the theorem is when $\left\{I_{i}\right\}$ are the closed reduced odd degree points on $X-R-\infty-o$, arranged by increasing degrees,

\footnotetext{
${ }^{1} \mathrm{~A}$ well-known fact from class field theory implies that the cardinality of $R$ is even and non-zero, and conversely, for any choice of a non-empty finite set $R \subset|X|$ of even cardinality there is a unique quaternion algebra ramified exactly at the places in $R$.
} 
and $H_{i}=Z\left(\mathcal{O}_{I_{i}}\right)$. In this case $X_{i}$ 's are the analogues of classical modular curves with full level structures along with a fixed choice of Weil pairing.

Remark 1.3. Unlike Drinfeld modular curves, which are affine, the modular curves of $\mathcal{D}$-elliptic sheaves are projective by construction, so one doesn't have to deal with the issue of the cusps.

1.3. Outline of the proof. Let $k$ be a finite field. Fix an algebraic closure $\bar{k}$ of $k$. In this subsection by a curve over $k$ we mean a proper, smooth scheme $C$ over $\operatorname{Spec}(k)$ of pure relative dimension 1 . We do not require a curve to be geometrically connected. The genus of $C$ is

$$
g(C):=\frac{1}{2} \operatorname{dim}_{\overline{\mathbb{Q}}_{\ell}} H_{e t}^{1}\left(C \otimes_{k} \bar{k}, \overline{\mathbb{Q}}_{\ell}\right),
$$

where $\ell$ is a prime different from the characteristic of $k$. If $C$ is geometrically connected then $g(C)$ is equal to the usual genus.

Let $C$ be a curve and let $G$ be a finite subgroup of $\operatorname{Aut}(C / k)$. The quotient scheme $C^{\prime}:=C / G$ is again a curve. The quotient morphism $\pi: C \rightarrow C^{\prime}$ is a Galois cover. Let $S$ be a finite subset of $C(\bar{k})$ and denote $S^{\prime}:=\pi(S) \subset C^{\prime}(\bar{k})$. Let $d$ be the degree of $\pi$. Since $g(C) \geq d\left(g\left(C^{\prime}\right)-1\right)$ and $\# S \leq d\left(\# S^{\prime}\right)$, there is an inequality

$$
\frac{\# S}{g(C)} \leq \frac{\# S^{\prime}}{g\left(C^{\prime}\right)-1}
$$

Now let $\mathcal{E} \ell \ell_{I, o}^{H}$ be as earlier, and let $\mathcal{E} \ell \ell_{I, o}\left(\overline{\mathbb{F}}_{o}\right)^{\text {ss }}$ be the set of super-singular points on $\mathcal{E} \ell \ell_{I, o}$ (see $\S 4$ for the definition). Using (1.1) and (1.2), we see that in order to prove the main theorem it is enough to prove the following four statements:

(1)

$$
\lim _{\operatorname{deg}(I) \rightarrow \infty} \frac{\# \mathcal{E} \ell \ell_{I, o}\left(\overline{\mathbb{F}}_{o}\right)^{\mathrm{ss}}}{g\left(\mathcal{E} \ell \ell_{I, o}\right)}=q_{o}-1 .
$$

(2) If $H$ contains $Z\left(\mathcal{O}_{I}\right)$ then the images of super-singular points on $\mathcal{E} \ell \ell_{I, o}^{H}$ are $\mathbb{F}_{o}^{(2)}$-rational.

(3) If $\operatorname{det}(H)$ generates $\mathcal{O}_{I}^{\times} / \mathbb{F}_{q}^{\times}$then $\mathcal{E} \ell \ell_{I, o}^{H}$ is geometrically connected.

(4) If $H \subseteq B\left(\mathcal{O}_{I}\right)$ then $g\left(\mathcal{E} \ell \ell_{I, o}^{H}\right) \rightarrow \infty$ as $\operatorname{deg}(I) \rightarrow \infty$.

Parts (1), (2) and (3) are proven as Propositions 5.1, 4.3 and 3.2, respectively. Part (4) easily follows by comparing the estimate (5.4) with the order of $B\left(\mathcal{O}_{I}\right)$.

\section{Notation}

Aside from the notation in Introduction, we will use the following:

2.1. For each closed point $x \in|X|$ (equivalently, a place of $F$ ), we denote by $\mathcal{O}_{x}$ and $F_{x}$ the completions of $\mathcal{O}_{X, x}$ and $F$ at $x$, respectively. The residue field of $\mathcal{O}_{x}$ is denoted by $\mathbb{F}_{x}$, the cardinality of $\mathbb{F}_{x}$ is denoted by $q_{x}$. We assume that the valuation $v_{x}: F_{x} \rightarrow \mathbb{Z}$ is normalized by $v_{x}\left(\varpi_{x}\right)=1$, where $\varpi_{x}$ is a uniformizer of $\mathcal{O}_{x}$; the norm $|\cdot|_{x}$ on $F_{x}$ is $q_{x}^{-v_{x}(\cdot)}$. We denote the adele ring of $F$ by $\mathbb{A}:=\prod_{x \in|X|}^{\prime} F_{x}$. For a set of places $S$ of $F$ we denote by $\mathbb{A}^{S}:=\prod_{x \notin S}^{\prime} F_{x}$ the adele ring outside $S$. 
2.2. Fix a locally free sheaf $\mathcal{D}$ of $\mathcal{O}_{X}$-algebras with stalk at the generic point equal to $D$ and such that $\mathcal{D}_{x}:=\mathcal{D} \otimes_{\mathcal{O}_{X}} \mathcal{O}_{x}$ is a maximal order in $D\left(F_{x}\right)$. Let $\mathcal{D}^{S}:=\prod_{x \notin S} \mathcal{D}_{x}$. For a finite nonempty closed subscheme $I$ of $X$, denote $\mathcal{D}_{I}=\mathcal{D} \otimes_{\mathcal{O}_{X}} \mathcal{O}_{I}$. Assuming $I$ is disjoint from $S$, let $K_{I}^{S}:=\operatorname{ker}\left(\left(\mathcal{D}^{S}\right)^{\times} \rightarrow \mathcal{D}_{I}^{\times}\right)$.

2.3. Let $\zeta_{F}(s)=\prod_{x \in|X|} \zeta_{x}(s)$ be the zeta function of $X$, where $\zeta_{x}(s)=\left(1-q_{x}^{-s}\right)^{-1}$. It is well-known (and is easy to show) that

$$
\zeta_{F}(s)=\frac{1}{\left(1-q^{-s}\right)\left(1-q^{1-s}\right)} .
$$

Let $\zeta_{F}^{S}(s)$ be the partial zeta function $\prod_{x \notin S} \zeta_{x}(s)$.

2.4. For a scheme $W$ over $\mathbb{F}_{q}$ denote by Frob ${ }_{W}$ its Frobenius endomorphism, which is the identity on the points and the $q$-th power map on the functions. We denote by $\operatorname{frob}_{q} \in \operatorname{Gal}\left(\overline{\mathbb{F}}_{q} / \mathbb{F}_{q}\right)$ the arithmetic Frobenius $\alpha \mapsto \alpha^{q}$, and for $x \in|X|$ we let $\operatorname{Frob}_{x}=\operatorname{frob}_{q}^{-\operatorname{deg}(x)} \in \operatorname{Gal}\left(\overline{\mathbb{F}}_{x} / \mathbb{F}_{x}\right)$ be the geometric Frobenius at $x$. Denote by $X \times W$ the fibred product $X \times_{\operatorname{Spec}\left(\mathbb{F}_{q}\right)} W$. For a sheaf $\mathcal{F}$ on $X$ and $\mathcal{G}$ on $W$, the sheaf $\operatorname{pr}_{1}^{*}(\mathcal{F}) \otimes \operatorname{pr}_{2}^{*}(\mathcal{G})$ on $X \times W$ is denoted by $\mathcal{F} \otimes \mathcal{G}$.

2.5. Let $D^{\times}$be the algebraic group over $F$ defined by $D^{\times}(B)=\left(D \otimes_{F} B\right)^{\times}$for any $F$-algebra $B$; this is the multiplicative group of $D$. Denote the algebra of $2 \times 2$ matrices by $\mathbb{M}_{2}$ and its multiplicative group $\mathrm{GL}_{2}$ by $G$.

\section{Modular curves of $\mathcal{D}$-elliptic sheaves}

Let $S$ be a $\mathbb{F}_{q}$-scheme, and let $z: S \rightarrow X$ be a morphism of $\mathbb{F}_{q}$-schemes such that $z(S) \subset X-R-\infty$. A $\mathcal{D}$-elliptic sheaf over $S$, with pole $\infty$ and zero $z$, is a sequence $\left(\mathcal{E}_{i}, j_{i}, t_{i}\right), i \in \mathbb{Z}$, where each $\mathcal{E}_{i}$ is a locally free sheaf of $\mathcal{O}_{X \times S}$-modules of rank 4 equipped with a right action of $\mathcal{D}$ compatible with the $\mathcal{O}_{X}$-action, and where

$$
\begin{aligned}
& j_{i}: \mathcal{E}_{i} \rightarrow \mathcal{E}_{i+1} \\
& t_{i}:{ }^{\tau} \mathcal{E}_{i}:=\left(\operatorname{id}_{X} \times \operatorname{Frob}_{S}\right)^{*} \mathcal{E}_{i} \rightarrow \mathcal{E}_{i+1}
\end{aligned}
$$

are injective $\mathcal{O}_{X \times S}$-linear homomorphisms compatible with $\mathcal{D}$-action. The maps $j_{i}$ and $t_{i}$ are sheaf modifications at $\infty$ and $z$, respectively, which satisfy certain conditions $[9, \S 2]$.

Let $I \neq \emptyset$ be a finite closed subscheme of $X-\infty$. Let $\left(\mathcal{E}_{i}, j_{i}, t_{i}\right)$ be a $\mathcal{D}$-elliptic sheaf over $S$ such that $z(S)$ is disjoint from $I$. Under this assumption, it turns out that the restrictions $\mathcal{E}_{I}:=\mathcal{E}_{i \mid I \times S}$, as well as the restrictions $t:=t_{i \mid I \times S}$, are independent of $i$, and $t$ induces an isomorphism ${ }^{\tau} \mathcal{E}_{I} \cong \mathcal{E}_{I}$. A level-I structure on $\left(\mathcal{E}_{i}, j_{i}, t_{i}\right)$ is an $\mathcal{O}_{I \times S}$-linear isomorphism $\iota: \mathcal{D}_{I} \otimes \mathcal{O}_{S} \cong \mathcal{E}_{I}$, compatible with the action of $\mathcal{D}_{I}$ on the right, which makes the following diagram commutative:

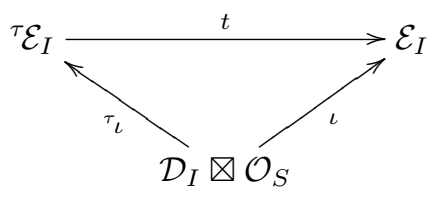


There is a natural action of $\mathbb{Z}$ on the category of $\mathcal{D}$-elliptic sheaves over $S$ with level- $I$ structures given by

$$
[n]\left(\mathcal{E}_{i}, j_{i}, t_{i}, \iota\right):=\left(\mathcal{E}_{i+n}, j_{i+n}, t_{i+n}, \iota\right) .
$$

For a scheme $z: S \rightarrow X^{\prime}:=X-\infty-I-R$, denote by $\mathbf{E l l}_{I}(S)$ the set of isomorphism classes of $\mathcal{D}$-elliptic sheaves over $S$ with zero $z$ and pole $\infty$, equipped with a level- $I$ structure, modulo the action of $\mathbb{Z}$. The first main theorem in [9] implies the following:

Theorem 3.1. The functor $S \mapsto \mathbf{E l l}_{I}(S)$ is representable by a smooth projective scheme $\mathcal{E} \ell \ell_{I}$ over $X^{\prime}$ of pure relative dimension 1.

The finite group $\mathcal{D}_{I}^{\times}$acts on $\mathcal{D}_{I} \otimes \mathcal{O}_{S}$ via right multiplication on $\mathcal{D}_{I}$. This induces an action of $\mathcal{D}_{I}^{\times}$on $\mathcal{E} \ell \ell_{I}$ via its action on the level structures. In terms of the moduli problem the action of $g \in \mathcal{D}_{I}^{\times}$is given by

$$
g:\left(\mathcal{E}_{i}, j_{i}, t_{i}, \iota\right) \mapsto\left(\mathcal{E}_{i}, j_{i}, t_{i}, \iota \circ g^{-1}\right) .
$$

Let $H$ be a subgroup of $\mathcal{D}_{I}^{\times}$. Denote the quotient of $\mathcal{E} \ell \ell_{I}$ under the action of $H$ by $\mathcal{E} \ell \ell_{I}^{H}$. Assume $I \cap R=\emptyset$. Then $\mathcal{D}_{I}^{\times} \cong G\left(\mathcal{O}_{I}\right)$. Suppose $H$ is such that

$$
\operatorname{det}: H \rightarrow \mathcal{O}_{I}^{\times} / \mathbb{F}_{q}^{\times},
$$

i.e., under the determinant homomorphism $H$ maps surjectively onto $\mathcal{O}_{I}^{\times} / \mathbb{F}_{q}^{\times}$.

Proposition 3.2. With above notation and assumptions, the fibres of the morphism $\mathcal{E} \ell \ell_{I}^{H} \rightarrow X^{\prime}$ are geometrically connected.

Proof. Let $\mathbb{C}_{\infty}$ be the completion of the algebraic closure of $F_{\infty}$. Consider the rigidanalytic variety $\mathcal{E} \ell \ell_{I}^{H}\left(\mathbb{C}_{\infty}\right)^{\text {an }}$. Denote by $\mathcal{H}^{\infty}$ the preimage of $H$ in $\left(\mathcal{D}^{\infty}\right)^{\times}$under the quotient map $\left(\mathcal{D}^{\infty}\right)^{\times} \rightarrow \mathcal{D}_{I}^{\times}$. By the theory of analytic uniformization [1, Thm. 4.4.11], the connected components of $\mathcal{E} \ell \ell_{I}^{H}\left(\mathbb{C}_{\infty}\right)^{\text {an }}$ are in one-to-one correspondence with

$$
D^{\times}(F) \backslash D^{\times}\left(\mathbb{A}^{\infty}\right) / \mathcal{H}^{\infty} \stackrel{\sim}{\longrightarrow} F^{\times} \backslash\left(\mathbb{A}^{\infty}\right)^{\times} / \operatorname{Nr}\left(\mathcal{H}^{\infty}\right),
$$

where the last isomorphism is induced by the reduced norm, and is a consequence of the strong approximation theorem. As is easy to check,

$$
F^{\times} \backslash\left(\mathbb{A}^{\infty}\right)^{\times} / \operatorname{Nr}\left(\mathcal{H}^{\infty}\right) \cong \mathbb{F}_{q}^{\times} \backslash \mathcal{O}_{I}^{\times} / \operatorname{det}(H) .
$$

Hence, under our assumption, $\mathcal{E} \ell \ell_{I}^{H}\left(\mathbb{C}_{\infty}\right)^{\text {an }}$ is connected. Since the number of connected components of an algebraic variety over $\mathbb{C}_{\infty}$ is equal to the number of connected components of the corresponding rigid-analytic space, we conclude that the generic fibre of $\mathcal{E} \ell \ell_{I}^{H}$ is geometrically connected. By Stein factorization theorem, all geometric fibres of $\mathcal{E} \ell \ell_{I}^{H} \rightarrow X^{\prime}$ are connected.

Remark 3.3. Theorem 4.4.11 of [1], which we used in the previous proof, is stated in the language of formal schemes; the desired description follows by applying Raynaud's "generic fibre" functor. It is unfortunate that the proof of this useful theorem is only vaguely outlined in [1]. Nevertheless, as is shown in [12], the desired uniformization can be deduced from Hausberger's version of the Cherednik-Drinfeld theorem for $\mathcal{E} \ell \ell_{I}$ $[6]$. 


\section{Super-singular $\mathcal{D}$-elliptic sheaves}

In this section we discuss a special class of $\mathcal{D}$-elliptic sheaves over extensions of $\mathbb{F}_{O}$, where $o \in|X|-\infty$.

One of the key preliminary results in [9] is the description of the points on the closed fibres of $\mathcal{E} \ell_{I} \rightarrow X^{\prime}$. This is done in two steps, similar to the description of the set of abelian varieties over finite fields: one starts by describing the isogeny classes of $\mathcal{D}$-elliptic sheaves and then parametrizes each isogeny class.

For the definition of isogenies between $\mathcal{D}$-elliptic sheaves over $k:=\overline{\mathbb{F}}_{o}$ we refer to $[9, \S 9]$. For our purposes it is enough to know [9, Thm. 9.13] that there is a canonical bijection between the set of isogeny classes of $\mathcal{D}$-elliptic sheaves over $k$ with zero $o$ and the pairs $(\widetilde{F}, \widetilde{\Pi})$ consisting of a finite separable field extension $\widetilde{F}$ of $F$, and an element $\widetilde{\Pi} \in \widetilde{F}^{\times} \otimes_{\mathbb{Z}} \mathbb{Q}$ which does not belong to $B^{\times} \otimes_{\mathbb{Z}} \mathbb{Q}$ for any proper $F$-subalgebra $B$ of $\widetilde{F}$. The pair $(\widetilde{F}, \widetilde{\Pi})$ must satisfy several technical conditions $[9,(9.11)]$. We say that $\left(\mathcal{E}_{i}, j_{i}, t_{i}\right)$ is super-singular if in its corresponding pair $(\widetilde{F}, \widetilde{\Pi})$ the field $\widetilde{F}$ is $F$ itself.

Lemma 4.1. All super-singular $\mathcal{D}$-elliptic sheaves are isogenous.

Proof. Extend the valuations from $F$ to $F^{\times} \otimes \mathbb{Q}$ by $v_{x}(f \otimes a)=a \cdot v_{x}(f)$. Then any element of $F^{\times} \otimes \mathbb{Q}$, up to an element of $\mathbb{F}_{q}^{\times}$, is uniquely determined by its valuations. If $\widetilde{F}=F$ then the valuations of $\widetilde{\Pi}$ by $[9,(9.11)]$ are $v_{\infty}(\widetilde{\Pi})=-1 / 2, v_{o}(\widetilde{\Pi})=1 / \operatorname{deg}(o) 2$, and $v_{x}(\widetilde{\Pi})=0$ if $x \neq o, \infty$. Therefore, up to an isomorphism, the pair $(\widetilde{F}, \widetilde{\Pi})$ is unique if $F=\widetilde{F}$.

From now on we assume that $I \subset X-R-o-\infty$ and $I \neq \emptyset$. Denote

$$
\mathcal{E} \ell \ell_{I, o}:=\mathcal{E} \ell_{I} \times_{X^{\prime}} \operatorname{Spec}\left(\mathbb{F}_{o}\right) .
$$

Let $\mathcal{E} \ell_{I, o}(k)^{\mathrm{ss}} \subset \mathcal{E} \ell_{I, o}(k)$ of the set of isomorphism classes of super-singular $\mathcal{D}$ elliptic sheaves over $k$ with level- $I$ structures. Let $\bar{D}$ be the quaternion algebra over $F$ with invariants

$$
\operatorname{inv}_{x} \bar{D}= \begin{cases}1 / 2, & \text { if } x=\infty \\ -1 / 2, & \text { if } x=o \\ \operatorname{inv}_{x}(D), & \text { otherwise }\end{cases}
$$

As a consequence of $[9, \S 10]$ and Lemma 4.1, we have

Theorem 4.2. There is a bijection

$$
\mathcal{E} \ell \ell_{I, o}(k)^{\mathrm{ss}} \stackrel{\sim}{\longrightarrow} \bar{D}^{\times}(F) \backslash\left[\left(\bar{D}^{\times}\left(\mathbb{A}^{\infty, o}\right) / K_{I}^{\infty, o} \times \mathbb{Z}\right],\right.
$$

where $\bar{D}^{\times}(F)$ is embedded diagonally into $\bar{D}^{\times}\left(\mathbb{A}^{\infty, o}\right)$, and its action on $\mathbb{Z}$ is via the composition of the reduced norm $\mathrm{Nr}$ with the valuation $v_{o}$. This bijection is compatible with the action of $\left(\mathcal{D}^{\infty, o}\right)^{\times}$. The action of $\mathrm{Frob}_{o}$ on $\mathcal{E} \ell_{I, o}(k)^{\mathrm{ss}}$ corresponds to the translation by 1 on $\mathbb{Z}$.

Let $H$ be a subgroup of $\mathcal{D}_{I}^{\times}$, and denote the quotient $\mathcal{E} \ell \ell_{I, o} / H$ by $\mathcal{E} \ell \ell_{I, o}^{H}$. Denote the preimage of $H$ in $\left(\mathcal{D}^{\infty, o}\right)^{\times}$by $\mathcal{H}^{\infty, o}$. Let $\mathcal{E} \ell \ell_{I, o}^{H}(k)^{\mathrm{ss}}$ be the image of $\mathcal{E} \ell \ell_{I, o}(k)^{\mathrm{ss}}$ under the quotient map. 
Proposition 4.3. If $Z\left(\mathcal{O}_{I}\right) \subseteq H$ then

$$
\mathcal{E} \ell \ell_{I, o}^{H}\left(\mathbb{F}_{o}^{(2)}\right)^{\mathrm{ss}}=\mathcal{E} \ell \ell_{I, o}^{H}(k)^{\mathrm{ss}},
$$

i.e., all the super-singular points on $\mathcal{E} \ell \ell_{I, o}^{H}$ are $\mathbb{F}_{o}^{(2)}$-rational.

Proof. As a consequence of Theorem 4.2, there is a bijection

$$
\mathcal{E} \ell \ell_{I, o}^{H}(k)^{\mathrm{ss}} \stackrel{\sim}{\longrightarrow} \bar{D}^{\times}(F) \backslash\left[\left(\bar{D}^{\times}\left(\mathbb{A}^{\infty, o}\right) / \mathcal{H}^{\infty, o} \times \mathbb{Z}\right],\right.
$$

and

$$
P=\bar{D}^{\times}(F)\left[g^{\infty, o} \mathcal{H}^{\infty, o}, m_{o}\right] \in \mathcal{E} \ell \ell_{I, o}^{H}(k)^{\mathrm{ss}}
$$

is rational over $\mathbb{F}_{o}^{(2)}$ if there is $\delta \in \bar{D}^{\times}(F)$ such that $\left(g^{\infty, o}\right)^{-1} \delta g^{\infty, o} \in \mathcal{H}^{\infty, o}$ and $v_{o}(\operatorname{Nr}(\delta))=2$. Let $f \in F^{\times}$be an element whose divisor on $X$ is $(f)=[o-\operatorname{deg}(o) \infty]$. Take $\delta=f \in F^{\times} \hookrightarrow \bar{D}^{\times}(F)$ as an element of the center of $\bar{D}^{\times}(F)$. Then $\operatorname{Nr}(f)=f^{2}$, and $v_{o}(\operatorname{Nr}(f))=2 \cdot v_{o}(f)=2$. We clearly have $f \in \mathcal{H}^{\infty, o}$, so $\left(g^{\infty, o}\right)^{-1} f g^{\infty, o}=f \in$ $\mathcal{H}^{\infty, o}$, and the first condition is also satisfied. Therefore, $P \in \mathcal{E} \ell \ell_{I, o}^{H}\left(\mathbb{F}_{o}^{(2)}\right)^{\mathrm{ss}}$. (Note that $f$ usually will not be in $K_{I}^{\infty, o}$.)

Now we compute the order of the set $\mathcal{E} \ell \ell_{I, o}(k)^{\mathrm{ss}}$. Fix a maximal order $\overline{\mathcal{D}}$ for $\bar{D}$ similar to $\mathcal{D}$ for $D$. Normalize the local multiplicative measure on $\bar{D}^{\times}\left(F_{x}\right)$ so that $\operatorname{Vol}\left(\overline{\mathcal{D}}_{x}^{\times}\right)=1$. Define the measure on $\bar{D}^{\times}(\mathbb{A})$ to be the restricted product measure. (As $\bar{D}^{\times}(\mathbb{A})$ and $\bar{D}^{\times}\left(F_{x}\right)$ are unimodular, we do not distinguish between left and right Haar measures.) Let $\bar{D}^{1}(\mathbb{A})$ be the kernel of the homomorphism $\|\cdot\|: \bar{D}^{\times}(\mathbb{A}) \rightarrow q^{\mathbb{Z}}$ given by the composition of the reduced norm $\mathrm{Nr}: \bar{D}^{\times}(\mathbb{A}) \rightarrow \mathbb{A}^{\times}$with the idelic norm $\prod_{x \in|X|}|\cdot|_{x}: \mathbb{A}^{\times} \rightarrow q^{\mathbb{Z}}$. The quotient $\bar{D}^{\times}(F) \backslash \bar{D}^{1}(\mathbb{A})$ is compact, hence has finite volume with respect to the push-forward measure. (Note that $\bar{D}^{\times}(F)$ lies in $\bar{D}^{1}(\mathbb{A})$, thanks to the product formula.)

\section{Proposition 4.4.}

$$
\# \mathcal{E} \ell \ell_{I, o}(k)^{\mathrm{ss}}=\frac{\# G\left(\mathcal{O}_{I}\right)\left(q_{o}-1\right)}{\left(q^{2}-1\right)(q-1)} \cdot \prod_{x \in R}\left(q_{x}-1\right) .
$$

Proof. Let $\bar{K}_{I}^{S}:=\operatorname{ker}\left(\left(\overline{\mathcal{D}}^{S}\right)^{\times} \rightarrow \overline{\mathcal{D}}_{I}^{\times}\right)$. By Theorem 4.2 ,

$$
\# \mathcal{E} \ell \ell_{I, o}(k)^{\mathrm{ss}}=\#\left(\bar{D}^{\times}(F) \backslash\left[\left(\bar{D}^{\times}\left(\mathbb{A}^{\infty, o}\right) / \bar{K}_{I}^{\infty, o} \times \mathbb{Z}\right]\right) .\right.
$$

(Note that $K_{I}^{\infty, o}=\bar{K}_{I}^{\infty, o}$.) Since $\bar{D}\left(F_{o}\right)$ is a division algebra, the maximal order $\overline{\mathcal{D}}_{o}^{\times}$is unique in $\bar{D}^{\times}\left(F_{o}\right)$ and is characterized by $\overline{\mathcal{D}}_{o}^{\times}=\left\{a \in \bar{D}^{\times}\left(F_{o}\right) \mid \operatorname{Nr}(a) \in \mathcal{O}_{o}^{\times}\right\}$; see [11, $\S 12]$. Hence the kernel of the reduced norm $\mathrm{Nr}$ on $\bar{D}^{\times}\left(F_{o}\right)$ is in $\overline{\mathcal{D}}_{o}^{\times}$. This, combined with the fact that $\mathrm{Nr}: \overline{\mathcal{D}}_{o}^{\times} \rightarrow \mathcal{O}_{o}^{\times}$is surjective, implies $v_{o} \circ \mathrm{Nr}: \bar{D}^{\times}\left(F_{o}\right) / \overline{\mathcal{D}}_{o}^{\times} \stackrel{\sim}{\longrightarrow} \mathbb{Z}$. Since $\bar{D}^{\times}(F)$ acts on $\mathbb{Z}$ in (4.1) via $v_{o} \circ \mathrm{Nr}$, we get

$$
\bar{D}^{\times}(F) \backslash\left[\left(\bar{D}^{\times}\left(\mathbb{A}^{\infty, o}\right) / \bar{K}_{I}^{\infty, o} \times \mathbb{Z}\right] \cong \bar{D}^{\times}(F) \backslash \bar{D}^{\times}\left(\mathbb{A}^{\infty}\right) / \bar{K}_{I}^{\infty} .\right.
$$

Since $\bar{D}\left(F_{\infty}\right)$ is a division algebra and $\infty$ is rational, for each $a \in \bar{D}^{\times}\left(\mathbb{A}^{\infty}\right)$, up to an element of $\overline{\mathcal{D}}_{\infty}^{\times}$, there is a unique $b \in \bar{D}^{\times}\left(F_{\infty}\right)$ such that $(a, b) \in \bar{D}^{1}(\mathbb{A})$. Hence,

$$
\bar{D}^{\times}(F) \backslash \bar{D}^{\times}\left(\mathbb{A}^{\infty}\right) / \bar{K}_{I}^{\infty} \cong \bar{D}^{\times}(F) \backslash \bar{D}^{1}(\mathbb{A}) / \bar{K}_{I} .
$$

Next, note that $\overline{\mathcal{D}}^{\times} / \bar{K}_{I} \cong G\left(\mathcal{O}_{I}\right)$. Since by our normalization of the measure we have $\operatorname{Vol}\left(\overline{\mathcal{D}}^{\times}\right)=1$, we conclude:

$$
\# \mathcal{E} \ell_{I, o}(k)^{\mathrm{ss}}=\# G\left(\mathcal{O}_{I}\right) \cdot \operatorname{Vol}\left(\bar{D}^{\times}(F) \backslash \bar{D}^{1}(\mathbb{A})\right) .
$$


It remains to compute the volume. This calculation is essentially contained in [15], but in absence of a convenient explicit reference we sketch some of the details.

One starts by relating the volume in question to the residue at 0 of a certain zeta function. Let $\Phi$ be the characteristic function of $\overline{\mathcal{D}}$ in $\bar{D}(\mathbb{A})$. Consider the following integral

$$
\zeta_{\bar{D}}(s)=\int_{\bar{D}^{\times}(\mathbb{A})} \Phi(z) \cdot\|z\|^{s} d^{\times} z .
$$

It absolutely converges for $\operatorname{Re}(s)>1$, can be meromorphically continued to the whole plane with a simple pole at 0 , and

$$
\operatorname{Res}_{s=0} \zeta_{\bar{D}}(s)=-\operatorname{Vol}\left(\bar{D}^{\times}(F) \backslash \bar{D}^{1}(\mathbb{A})\right) \frac{1}{\log q} .
$$

Next, we have the decomposition $\zeta_{\bar{D}}(s)=\prod_{x} \zeta_{\bar{D}_{x}}(s)$, where

$$
\zeta_{\bar{D}_{x}}(s)=\int_{\bar{D}^{\times}\left(F_{x}\right)} \Phi_{x}\left(z_{x}\right) \cdot\left|\operatorname{Nr}\left(z_{x}\right)\right|_{x}^{s} d^{\times} z_{x} .
$$

If $\bar{D}$ is split at $x$ then, by considering the decomposition of $\mathbb{M}_{2}\left(\mathcal{O}_{x}\right)$ into left $\overline{\mathcal{D}}_{x}^{\times} \cong$ $G\left(\mathcal{O}_{x}\right)$-cosets, we get

$$
\begin{aligned}
\zeta_{\bar{D}_{x}}(s) & =\int_{\mathbb{M}_{2}\left(\mathcal{O}_{x}\right)-\{0\}}\left|\operatorname{Nr}\left(z_{x}\right)\right|_{x}^{s} d^{\times} z_{x} \\
& =\operatorname{Vol}\left(\overline{\mathcal{D}}_{x}^{\times}\right)\left(\sum_{n=0}^{\infty} q_{x}^{-s n}\right)\left(\sum_{n=0}^{\infty} q_{x}^{(-s+1) n}\right)=\zeta_{x}(s) \cdot \zeta_{x}(s-1) .
\end{aligned}
$$

On the other hand, if $\bar{D}$ is ramified at $x$ then $\bar{D}\left(F_{x}\right)$ is a division algebra, hence $\overline{\mathcal{D}}_{x}$ has a unique maximal ideal $\mathfrak{P} \triangleleft \overline{\mathcal{D}}_{x}$ and $\operatorname{Nr}(\mathfrak{P})=\varpi_{x}$; see [11, (24.13)]. Thus,

$$
\zeta_{\bar{D}_{x}}(s)=\int_{\overline{\mathcal{D}}_{x}-\{0\}}\left|\operatorname{Nr}\left(z_{x}\right)\right|_{x}^{s} d^{\times} z_{x}=\operatorname{Vol}\left(\overline{\mathcal{D}}_{x}^{\times}\right) \sum_{n=0}^{\infty} q_{x}^{-s n}=\zeta_{x}(s) .
$$

Let $S=R \cup\{\infty, o\}$. Combining the local calculations,

Therefore

$$
\operatorname{Res}_{s=0} \zeta_{\bar{D}}(s)=-\frac{1}{(q-1) \log q} \cdot \zeta_{F}^{S}(-1)
$$

$$
\operatorname{Vol}\left(\bar{D}^{\times}(F) \backslash \bar{D}^{1}(\mathbb{A})\right)=\frac{\left(q_{o}-1\right)}{\left(q^{2}-1\right)(q-1)} \cdot \prod_{x \in R}\left(q_{x}-1\right),
$$

and the proposition follows.

\section{Genus calculations}

Let $\mathcal{A}\left(D^{\times}(F) \backslash D^{\times}(\mathbb{A}) / \varpi_{\infty}^{\mathbb{Z}}\right)$ be the space of $\overline{\mathbb{Q}}_{\ell^{-v a l u e d}}$ locally constant functions on $D^{\times}(\mathbb{A}) / \varpi_{\infty}^{\mathbb{Z}}$ which are invariant under the action of $D^{\times}(F)$ on the left. This space is equipped with the right regular representation of $D^{\times}(\mathbb{A}) / \varpi_{\infty}^{\mathbb{Z}}$. Since $D$ is a division algebra, the coset space $D^{\times}(F) \backslash D^{\times}(\mathbb{A}) / \varpi_{\infty}^{\mathbb{Z}}$ is compact and decomposes as a sum of irreducible admissible representations

$$
\mathcal{A}\left(D^{\times}(F) \backslash D^{\times}(\mathbb{A}) / \varpi_{\infty}^{\mathbb{Z}}\right)=\bigoplus_{\Pi} \Pi
$$


where each representation appears with multiplicity one, cf. $[9, \S 13]$. The representations appearing in this sum are called automorphic.

Each automorphic representation $\Pi$ decomposes as a restricted tensor product $\Pi=\otimes_{x \in|X|} \Pi_{x}$ of admissible irreducible representations of $D^{\times}\left(F_{x}\right)$. We denote $\Pi^{\infty}=$ $\otimes_{x \neq \infty} \Pi_{x}$, so $\Pi=\Pi^{\infty} \otimes \Pi_{\infty}$. If $\Pi$ is finite dimensional, then it is of the form $\Pi=\chi \circ \mathrm{Nr}$, where $\chi$ is a Hecke character of $\mathbb{A}^{\times}$, cf. $[9$, Lem. 14.8]. If $\Pi$ is infinite dimensional, then $\Pi_{x}$ is infinite dimensional for every $x \notin R$.

Let $\psi$ be a character of $F_{x}^{\times}$. Denote by $\operatorname{Sp}_{x} \otimes \psi$ the unique irreducible quotient of the induced representation

$$
\operatorname{Ind}_{B}^{G}\left(|\cdot|_{x}^{-\frac{1}{2}} \psi \oplus|\cdot|_{x}^{\frac{1}{2}} \psi\right) .
$$

The representation $\mathrm{Sp}_{x} \otimes \psi$ is called the special representation of $G\left(F_{x}\right)$ twisted by $\psi$. If $\psi=1$, then we simply write $\mathrm{Sp}_{x}$.

Let $\eta=\operatorname{Spec}(F)$ be the generic point of $X$ and fix an algebraic closure $\bar{F}$ of $F$. Let $\mathcal{E} \ell \ell_{I, \eta}:=\mathcal{E} \ell \ell_{I} \times_{X} \operatorname{Spec}(F)$. Fix a prime number $\ell \neq p$ and consider the $\ell$-adic cohomology groups $H_{I, \eta}^{i}:=H^{i}\left(\mathcal{E} \ell \ell_{I, \eta} \otimes_{F} \bar{F}, \overline{\mathbb{Q}}_{\ell}\right)$. These are finite dimensional $\overline{\mathbb{Q}}_{\ell}$-vector spaces, which are non-zero only if $0 \leq i \leq 2$.

Denote by $W\left(D^{\times} ; I\right)$ the subspace of $\mathcal{A}\left(D^{\times}(F) \backslash D^{\times}(\mathbb{A}) / \varpi_{\infty}^{\mathbb{Z}}\right)$ consisting of automorphic representations $\Pi$ such that the subspace of $K_{I}^{\infty}$-fixed vectors $\left(\Pi^{\infty}\right)^{K_{I}^{\infty}}$ is non-zero and $\Pi_{\infty} \cong \mathrm{Sp}_{\infty}$. (Keep in mind that $D^{\times}\left(F_{\infty}\right) \cong G\left(F_{\infty}\right)$ since by assumption $D$ is split at $\infty$.)

Taking the $K_{I}^{\infty}$-invariants in Theorems 14.9 and 14.12 of [9], we get

$$
\operatorname{dim}_{\overline{\mathbb{Q}}_{\ell}} H_{I, \eta}^{1}=2 \sum_{\Pi \in W\left(D^{\times} ; I\right)} \operatorname{dim}_{\overline{\mathbb{Q}}_{\ell}}\left(\Pi^{\infty}\right)^{K_{I}^{\infty}} .
$$

Let $H_{I, o}^{1}:=H^{1}\left(\mathcal{E} \ell \ell_{I, o} \otimes_{\mathbb{F}_{o}} \overline{\mathbb{F}}_{o}, \overline{\mathbb{Q}}_{\ell}\right)$. By proper base change theorem, there is a canonical isomorphism $H_{I, o}^{1} \cong H_{I, \eta}^{1}$ of $\overline{\mathbb{Q}}_{\ell}$-vector spaces. Hence

$$
g\left(\mathcal{E} \ell \ell_{I, o}\right)=\sum_{\Pi \in W\left(D^{\times} ; I\right)} \operatorname{dim}_{\overline{\mathbb{Q}}_{\ell}}\left(\Pi^{\infty}\right)^{K_{I}^{\infty}} .
$$

To estimate this last sum, we transfer the question from $D^{\times}$to $G$. The global Jacquet-Langlands correspondence $[8, \mathrm{Ch}$. III] associates to each infinite dimensional automorphic representation $\Pi$ of $D^{\times}(\mathbb{A})$ a cuspidal representation $\Pi^{\prime}=\operatorname{JL}(\Pi)$ of $G(\mathbb{A})$ with the following properties:

(1) if $x \notin R$ then $\Pi_{x} \cong \Pi_{x}^{\prime}$;

(2) if $x \in R$ and $\Pi_{x} \cong \psi \circ \mathrm{Nr}$ for a character $\psi$ of $F_{x}^{\times}$, then

$$
\Pi_{x}^{\prime} \cong \mathrm{Sp}_{x} \otimes \psi \text {. }
$$

Suppose $\Pi^{\infty}$ has a non-zero $K_{I}^{\infty}$-fixed vector. Then, for $x \in R, \Pi_{x}$ has a non-zero vector fixed by $\mathcal{D}_{x}^{\times}$(since $I \cap R=\emptyset$ by assumption). On the other hand, $\mathcal{D}_{x}^{\times}$is normal in $D^{\times}\left(F_{x}\right)$ and $D^{\times}\left(F_{x}\right) / \mathcal{D}_{x}^{\times} \cong \mathbb{Z}$. Therefore, $\Pi_{x}=\psi \circ \mathrm{Nr}$ for some unramified character of $F_{x}^{\times}$( $\psi$ is unramified because the reduced norm maps $\mathcal{D}_{x}^{\times}$surjectively onto $\left.\mathcal{O}_{x}^{\times}\right)$. Thus, for $x \in R, \Pi_{x}^{\prime}$ is a twist of $\mathrm{Sp}_{x}$ by an unramified character.

Let $J_{x}$ be the Iwahori subgroup of $G\left(\mathcal{O}_{x}\right)$, i.e., the subgroup of matrices which maps to $B\left(\mathbb{F}_{x}\right)$ modulo $\varpi_{x}$. The representations of the form $\operatorname{Sp}_{x} \otimes \psi$, with $\psi$ unramified, 
can be characterized by the property that they have a unique 1-dimensional $J_{x}$-fixed subspace; see [2]. For a finite set $S \subset|X|-\infty$ define a subgroup of $G\left(\mathbb{A}^{\infty}\right)$

$$
J_{S}^{\infty}:=\prod_{x \in S} J_{x} \prod_{x \in|X|-S-\infty} G\left(\mathcal{O}_{x}\right) .
$$

Denote by $W(G ; I, R)$ the set of cuspidal representations of $G(\mathbb{A})$ which have a non-zero $K_{I}^{\infty} \cap J_{R}^{\infty}$-fixed vector and $\infty$ component isomorphic to $\mathrm{Sp}_{\infty}$. Define the $R$-new part $W(G ; I, R)^{R \text {-new }}$ of $W(G ; I, R)$ to be the representations which do not have a non-zero $K_{I}^{\infty} \cap J_{S}^{\infty}$-fixed vector for any $S$ strictly contained in $R$. From what was said, we know that the image of $W\left(D^{\times} ; I\right)$ under JL lies in the $R$-new part of $W(G ; I, R)$, and in fact, as part of the theory, it is known that the Jacquet-Langlands correspondence gives a bijection

$$
\mathrm{JL}: W\left(D^{\times} ; I\right) \stackrel{\sim}{\longrightarrow} W(G ; I, R)^{R-\text { new }} .
$$

One can think of $R$ as a reduced closed subscheme of $X-\infty$. Write $I+R$ for the scheme-theoretic union of $I$ and $R$. Note that $W(G ; I, R)$ makes sense for an arbitrary finite $R$, so from now on $R$ is an arbitrary closed subscheme of $X-\infty$, disjoint from $I$. To simplify the notation, we write $W(I, R)$ for $W(G ; I, R)$, and $W(I)$ for $W(G ; I, \emptyset)$. Let

$$
w(I, R):=\sum_{\Pi \in W(R, I)} \operatorname{dim}_{\overline{\mathbb{Q}}_{\ell}}\left(\Pi^{\infty}\right)^{K_{I}^{\infty} \cap J_{R}^{\infty}} .
$$

Similar notation will be used for sums over subsets of $W(I, R)$, e.g., $w(I, R)^{R-\text { new }}$ is the sum over $\Pi$ in $W(I, R)^{R-\text { new }}$.

The number $w(I)$ is well-known and can be computed in several different (although interrelated) ways. For example, one can use Drinfeld's fundamental theorem [3, Thm. 2 ] and relate $w(I)$ to the genus of the modular curve $X(I)$ classifying rank-2 Drinfeld modules over $F$ with full level- $I$ structure. Since we are mostly concerned with the asymptotic behavior, for simplicity we only give the asymptotic formulae.

Write $f(I) \asymp g(I)$ for two $\mathbb{Q}$-valued functions $f(I)$ and $g(I)$ depending on $I$, if $f(I) / g(I) \rightarrow 1$ as $\operatorname{deg}(I) \rightarrow \infty$. Then one has the following (see Theorem 8.1 in [5])

$$
w(I) \asymp \frac{\# G\left(\mathcal{O}_{I}\right)}{\left(q^{2}-1\right)(q-1)} .
$$

Now $w(I, R)$ can be deduced from $w(I+R)$ :

$$
w(I, R) \asymp \frac{\# G\left(\mathcal{O}_{I}\right)}{\left(q^{2}-1\right)(q-1)} \prod_{x \in R}\left(q_{x}+1\right) .
$$

Geometrically this can be seen as follows. Let $X(I, R)$ be the modular curve classifying rank-2 Drinfeld modules over $F$ with a full level- $I$ structure and a cyclic level- $R$ structure. There is a natural Galois covering $X(I+R) \rightarrow X(I, R)$ with Galois group $B\left(\mathcal{O}_{R}\right)$. If $I$ is large enough then this covering is étale, so the genus of $X(I, R)$ is asymptotically equal to the genus of $X(I+R)$ divided by $\# B\left(\mathcal{O}_{R}\right)$. Now note that

$$
\# G\left(\mathcal{O}_{I+R}\right) / \# B\left(\mathcal{O}_{R}\right)=\# G\left(\mathcal{O}_{I}\right) \prod_{x \in R}\left(q_{x}+1\right) .
$$

For a subset $P \subseteq R$ define $W(I, R)^{P-\text { new }}$ to be the subset of $W(I, R)$ consisting of representations which do not have a $K_{I}^{\infty} \cap J_{S}^{\infty}$-fixed vector for any $S$ strictly contained 
in $P$. Let $x \in R$ be a place, and write $R=R^{\prime}+x$. Since the Atkin-Lehner theory of new forms on $G$ works over $F$ essentially the same way as for classical cusp forms, we have

$$
W(I, R)=W(I, R)^{x-\text { new }} \oplus W(I, R)^{x-\text { old }},
$$

where

$$
W(I, R)^{x-\text { old }} \cong W\left(I, R^{\prime}\right) \oplus W\left(I, R^{\prime}\right) .
$$

Hence

$$
w(I, R)^{x-\text { new }}=w(I, R)-2 w(I, R-x) .
$$

Next, let $x, z \in R$ be two distinct places and write $R=R^{\prime}+x+z$. Then

$$
W(I, R)^{x-\text { old }} \cap W(I, R)^{z-\text { old }} \cong W\left(I, R^{\prime}\right)^{\oplus 4},
$$

and, using the exclusion-inclusion principle, we get

$$
w(I, R)^{\{x, z\}-\text { new }}=w(I, R)-2 w(I, R-x)-2 w(I, R-z)+4 w(I, R-x-z) .
$$

Expanding this argument,

$$
w(I, R)^{R-\text { new }}=\sum_{P \subseteq R}(-2)^{\# P} w(I, R-P),
$$

where the sum is over all subsets of $R$. Finally, we can combine this with (5.3) to obtain

$$
\begin{aligned}
w(I, R)^{R-\text { new }} \asymp & \frac{\# G\left(\mathcal{O}_{I}\right)}{\left(q^{2}-1\right)(q-1)}\left(\sum_{P \subseteq R}(-2)^{\# P} \prod_{x \in R-P}\left(q_{x}+1\right)\right) \\
& =\frac{\# G\left(\mathcal{O}_{I}\right)}{\left(q^{2}-1\right)(q-1)} \prod_{x \in R}\left(q_{x}-1\right) .
\end{aligned}
$$

This estimate, combined with (5.1) and (5.2), gives the following asymptotic formula:

$$
g\left(\mathcal{E} \ell \ell_{I, o}\right) \asymp \frac{\# G\left(\mathcal{O}_{I}\right)}{\left(q^{2}-1\right)(q-1)} \prod_{x \in R}\left(q_{x}-1\right) .
$$

\section{Proposition 5.1.}

$$
\lim _{\operatorname{deg}(I) \rightarrow \infty} \frac{\# \mathcal{E} \ell \ell_{I, o}\left(\overline{\mathbb{F}}_{o}\right)^{\mathrm{ss}}}{g\left(\mathcal{E} \ell \ell_{I, o}\right)}=q_{o}-1 .
$$

Proof. This follows from Proposition 4.4 and (5.4).

\section{References}

[1] A. Blum and U. Stuhler, Drinfeld modules and elliptic sheaves, in Vector bundles on curves: New directions, LNM 1649, Springer (1997) 110-188.

[2] W. Casselman, On some results of Atkin and Lehner, Math. Ann. 201 (1973) 301-314.

[3] V. Drinfeld, Elliptic modules, Math. Sbornik 94 (1974) 594-627.

[4] N. Elkies, Explicit modular towers, arXiv:math.NT/0103107v1 (2001)

[5] E.-U. Gekeler, Invariants of some algebraic curves related to Drinfeld modular curves, J. Number Theory 90 (2001) 166-183.

[6] T. Hausberger, Uniformisation des variétés de Laumon-Rapoport-Stuhler et conjecture de Drinfeld-Carayol, Ann. Inst. Fourier 55 (2005) 1285-1371.

[7] Y. Ihara, Some remarks on the number of rational points of algebraic curve over finite fields, J. Fac. Sci. Univ. Tokyo 28 (1981) 721-724.

[8] H. Jacquet and R. Langlands, Automorphic forms on GL(2), LNM 114, Springer (1970). 
[9] G. Laumon, M. Rapoport, and U. Stuhler, D-elliptic sheaves and the Langlands correspondence, Invent. Math. 113 (1993) 217-338.

[10] Y. Manin and S. Vladut, Linear codes and modular curves, J. Sov. Math. 30 (1985) 2611-2643.

[11] I. Reiner, Maximal orders, Academic Press (1975).

[12] M. Spiess, Twists of Drinfeld-Stuhler modular varieties, arXiv:math.AG/0701566v1 (2007)

[13] M. Tsfasman, S. Vladut, and T. Zink, Modular curves, Shimura curves, and Goppa codes, better than the Varschamov-Gilbert bound, Math. Nachr. 109 (1982) 21-28.

[14] S. Vladut and V. Drinfeld, The number of points of an algebraic curve, Funct. Anal. Appl. 17 (1983) 53-54.

[15] A. Weil, Adeles and algebraic groups, Progress in Math. 23, Birkhäuser (1982).

Department of Mathematics, Pennsylvania State University, University Park, PA 16802

E-mail address: papikian@math.psu.edu 